\title{
MÉDIATION ET MISE EN SCÈNE DE L'INFORMATION DANS LE DISCOURS DE VULGARISATION SCIENTIFIQUE
}

\begin{abstract}
Sabban Annette, Médiation et mise en scène de l'information dans le discours de vulgarisation scientifique [The mamer of presentation of information in the texts popularising science]. Studia Romanica Posnaniensia, Adam Mickiewicz University Press, Poznań, vol. XXV/XXVI: 2000, pp. 269281, ISBN 83-232-0965-0, ISSN 0137-2475.

The present study examines texts about computer viruses from French, Spanish and German popular scientific journals. The first part of the paper shows how these texts make systematic use of concepts and expressions drawn from other domains of discourse. The second part of the paper focusses on a subgroup of the linguistic means employed, i.e. metaphorical expressions, and discusses their functions in the texts under discussion. These functions can be assigned to two groups, in that they either mediate new information or make the message more effective on the rhetorical level. The paper concludes with a discussion of some writer strategies used for activating other semantic domains as a prerequisite for achieving a particular thetorical effect.
\end{abstract}

\section{INTRODUCTION}

Dans cet article, il sera question d'un certain type de discours, à savoir le discours de vulgarisation scientifique. Plus précisément, $\mathrm{j}$ 'examinerai la façon dont un thème précis ${ }^{1}$ est traité à l'intérieur de ce type de discours. Le thème que j'ai choisi, c'est le virus informatique.

\subsection{LE VIRUS INFORMATIQUE}

En quoi ce sujet peut-il intéresser le linguiste? Les virus informatiques - on le sait bien - sont un phénomène assez récent: ils sont apparus au début des années 80 , à la suite de la 'révolution informatique' ${ }^{2}$. Nous sommes donc en présence d'un cas

${ }^{1}$ Le thème du texte est ce qui en fait l'objet et qui correspond à l'allemand Textthema ou bien à l'anglais discourse topic. - Je voudrais remercier Monique Krötsch pour ses remarques critiques ainsi que Michel Lachaud pour la relecture de cet article.

${ }^{2}$ La première publication scientifique sur les virus informatiques date de 1985 et a eu lieu aux Etats-Unis. L'expression virus informatique est très certainement née à cette époque-là; cf. Brunnstein 1991: 30. 
exemplaire dans lequel un phénomène nouveau, ainsi que tout un ensemble de phénomènes qui y sont rattachés, doivent être maîtrisés linguistiquement, c'est-à-dire qu'ils doivent être définis conceptuellement et désignés.

\subsection{LE DISCOURS DE VULGARISATION SCIENTIFIQUE}

Sur le fond, le fonctionnement de "ces virus du troisième type» ${ }^{3}$ relève du domaine de l'informatique, car il s'agit d'une 'anomalie programmée' du logiciel, donc d'une anomalie provoquée délibérément. Or, en général, les détails techniques de la programmation n'intéressent pas l'utilisateur de l'ordinateur. Celui-ci voudrait plutôt se faire une idée du mode de fonctionnement de ces virus afin de s'en protéger et de prendre des mesures appropriées. Ces informations, il peut se les procurer dans un des nombreux guides qu'il y a sur le marché ${ }^{4}$, il peut les trouver dans les revues de vulgarisation scientifique ${ }^{5}$ ou même aux pages correspondantes des quotidiens. Les textes produits par ces publications relèvent tous du discours de vulgarisation scientifique $^{6}$ : ils présentent un contenu technique ou scientifique tout en s'adressant à un public de non-spécialistes. Le rôle de l'auteur du texte - le plus souvent un journaliste - est double: d'une part, il est un «communicateur professionnel» entre spécialistes et non-spécialistes (Loffler-Laurian 1994) qui désire transmettre des informations sur des contenus spécialisés ${ }^{7}$. D'autre part, en tant que journaliste, il veut aussi susciter l'intérêt du lecteur, rendre son texte intéressant, divertissant - bref, il veut 'mettre en scène' l'information ${ }^{8}$. Par discours de vulgarisation scientifique, j'entends donc l'ensemble des textes s'insérant dans ce cadre général.

\section{OBJECTIFS}

Mes objectifs sont de deux ordres:

1. Caractériser certains moyens linguistiques utilisés dans le discours sur les virus informatiques. J'appelle ce discours discours cible. Plus précisément, je voudrais montrer comment ce discours cible est construit systématiquement par le moyen d'expressions provenant d'autres discours que j'appellerai discours sources.

${ }^{3}$ La Recherche 280, oct. 1995: 24; PCmag 111, juin 1997: 125.

${ }^{4}$ Voir Lilen 1996 (français), de Marcelo Rodao 1995 (espagnol), Brunnstein 1991 (allemand).

${ }^{5}$ Pour la France voir par exemple Sciences et Avenir ou La Recherche; pour l'Espagne Conocer, Natura ou bien Muy interesante. A part ces revues de vulgarisation 'générales', il existe des revues plus spécialisées qui s'adressent au lecteur plus ou moins au fait de la micro-informatique; les revues dont sont tirées les textes exploités ici (pour les détails voir l'annexe).

${ }^{6}$ Pour l'histoire de cette désignation, remplacée de plus en plus souvent par d'autres termes tels que communication ou information scientifique pour éviter l'évocation de connotations péjoratives, voir Jeanneret 1994: 15 ss.

${ }^{7}$ Pour une définition du discours de vulgarisation scientifique voir aussi Mortureux 1994: 3, 8 et Fernández Sánchez 1990: 287.

${ }^{8}$ Il faut ajouter que c'est aussi une caractéristique de la médiatisation actuelle qui met souvent en valeur l'aspect ludique, même pour les contenus spécialisés ou les reportages politiques. 
Les notions de discours source et discours cible rappellent celles de langue source et langue cible en traductologie. Il s'agit effectivement d'une sorte de traduction, mais qui s'effectue à l'intérieur d'une même langue'.

Les expressions 'transposées' d'un discours à l'autre peuvent être des lexèmes ou bien des unités plus larges, comme les collocations. Un sous-ensemble important, parmi ces expressions, est constitué par les métaphores, ou plutôt par des champs métaphoriques entiers ${ }^{10}$. Par champ métaphorique, j'entends un ensemble de métaphores qui 'puisent' à un même domaine source pour désigner des concepts dans un même domaine cible ${ }^{11}$.

Les métaphores sont particulièrement aptes à remplir les deux fonctions mentionnées plus haut: celle de rendre le message intelligible, d'ouvrir au lecteur, pour ainsi dire, une voie d'accès cognitive, et celle de mettre en scène l'information dont il est question. Dans ce qui suit, je mettrai l'accent sur les métaphores, mais il est important de saisir d'emblée que celles-ci ne sont que les 'exposants' d'un processus plus large.

2. Deuxième objectif: préciser les facteurs contribuant à déterminer la fonction d'une métaphore dans un contexte donné. Ces facteurs s'avèrent avant tout d'ordre discursif, mais nous verrons qu'ils sont aussi d'ordre linguistique au sens plus étroit.

\section{MÉTHODE ET ANALYSE}

J'ai dépouillé un certain nombre de textes traitant du même sujet et relevant du même type de discours (pour les détails voir la bibliographie). J'ai noté toutes les métaphores dans leurs contextes ainsi que les expressions non métaphoriques qui m'ont paru typiques d'un certain discours source. J'ai établi ainsi près de trois cents fiches que j'ai ensuite essayé d'ordonner. Le classement le plus révélateur m'a paru d'ordre thématique, c'est-à-dire groupant les exemples par sous-thèmes caractéristi-

${ }^{9}$ Cf. le concept de intralinguale Übersetzung ('traduction à l'intérieur d'une même langue') utilisé par Koller 1992: 283. Le rôle de l'auteur d'un texte de vulgarisation peut être comparé à celui du traducteur dans la mesure où tous deux essaient de rendre intelligible un message à l'aide d'un répertoire de moyens linguistiques qui sont (ou: sont censés être) à la disposition du destinataire envisagé. - Pour éviter un élargissement de la notion de traduction impliqué par un tel emploi du terme, mieux vaut parler d'une activité discursive de reformulation, comme le fait Mortureux 1994: 3; voir aussi Moirand 1994: 1.

${ }^{10}$ Rappel: La raison en est le fonctionnement de la métaphore. Celui-ci repose sur l'établissement d'une analogie entre un concept A, qui est connu (ou supposé tel), et certains aspects d'un concept B, qui, lui, est nouveau - soit qu'il s'agisse vraiment d'un concept inconnu au récepteur (ou supposé tel), soit que le locuteur veuille présenter un concept connu dans une nouvelle perspective pour changer le mode de concevoir un objet ou phénomène.

${ }^{11}$ Cf. Lakoff 1987: 288, qui pourtant, n'utilise pas la notion de champ métaphorique. Celle-ci est empruntée à Weinrich 1976: 283 s. - L'observation que les métaphores ne sont pas des curiosités isolées mais qu'elles sont souvent reliées entre elles a été le point de départ pour la linguistique cognitive aux Etats-Unis au début des années 80 (voir Lakoff/Johnson 1980). Cependant, il y a lieu de souligner que des observations semblables avaient été faites par Weinrich bien avant cette date. 
ques de ces textes. Ce classement permet de prendre conscience de toute la gamme des possibilités de formulation. C'est ce mode que j'utiliserai pour présenter quelques-uns des résultats.

\section{I. SOUS-THÈME 1: LE MODE D'ACTION DES VIRUS}

Comment est décrit le mode d'action des virus? Du point de vue technique, la première action du virus est de copier son code sur le disque dur, dans un fichier, etc. Ce processus est décrit par des expressions métaphoriques provenant de deux discours sources différents.

\section{DISCOURS SOURCE 1: LA MÉDECINE}

Le verbe infecter occupe une place centrale. On retrouve des équivalents immédiats dans les autres langues, construits de manière identique, c'est-à-dire avec des actants équivalents ${ }^{12}$.

(1) le virus infecte le logiciel (cf. Le Nouvel Observateur 28/4/94: 48; Le Monde 6/2/1996: 1),

(2) des documents infectés par des virus connus (PCmag juin 1997: 132),

(3) l'ordinateur infecté (Le Monde 6/2/1996: 1),

(4) los programas que no han sido infectados (PC actual, abril 1991: 220),

(5) los ficheros/ordenadores infectados (de Marcelo Rodao 1995: 173; 66),

(6) los virus pasan a la acción e infectan el ordenador (PC actual, abril 1991: 221),

(7) Der Virus [...] infiziert Programme (Brunnstein 1991: 68),

(8) das Software-Haus hat andere Anwenderprogramme mit dem Virus infiziert (ibid.: 32),

En y regardant de plus près, on constate que ce n'est pas un verbe isolé (soit: infecter et ses équivalents) mais toute une collocation sujet-verbe qui est, pour ainsi dire, transposée du discours médical dans le discours informatique. Cette collocation, c'est le virus qui infecte un OBJET.

\section{DISCOURS SOURCE 2: LA GUERRE}

Le mode d'action du virus est décrit par des expressions concurrentes relevant du domaine de la guerre. Ce sont avant tout les verbes attaquer ou s'attaquer, et les verbes correspondants dans les autres langues qui remontent tous à la même origine étymologique (esp. atacar, all. attackieren). On trouve aussi des substantifs de la

\footnotetext{
${ }^{12}$ Parfois, les actants sont reliés par métonymie, p. ex. Virus - Software-Haus dans (7) et (8), reliés selon le principe métonymique 'produit - producteur'.
} 
même famille de mots ou bien des expressions synonymes (fr. attaque, agression; esp. ataque).

(9) les virus s'attaquent aux informations du disque dur (PC direct 10/1996:140; voir aussi Lilen 1996: XIV),

(10) une attaque virale (Lilen 1996: XV),

(11) faire face à cette agression moderne (ibid.: XIV),

(12) el virus ataca al ordenador (PC actual, abril 1991: 222),

(13) estos virus que atacan a las máquinas (ibid.: 221),

(14) el ordenador bajo ataque (de Marcelo Rodao 1995: X),

(15) Der Michelangelo-Virus attackiert die Festplatte (Lübecker Nachrichten 8/6/96: 5),

J'ai distingué jusqu'ici deux discours sources. Si l'on considère d'autres sous-thèmes des textes, on constate nombre de métaphores puisant à ces mêmes discours sources. Mais le rapport entre discours sources et discours cibles ne se réduit pas aux métaphores. Dans ce qui suit, j'aimerais en donner une courte illustration.

\subsection{SOUS-THÈME 2: LES CONTRE-MESURES}

DISCOURS SOURCE 1: LA MÉDECINE

Dans la description des contre-mesures sont envisagées des mesures d'ordre préventif et d'ordre curatif. On retrouve donc la mềme catégorisation qu'en médecine:

(16) méthodes préventives et curatives inefftcaces (Lilen 1996: 2),

(17) Prévenir y guérir les macrovirus (titre de paragraphe, PCmag juin 1997: 130),

(18) Prevenir y curar (titre de paragraphe, PC actual, abril 1991: 221),

(19) Sistemas de prevención (titre de chapitre, de Marcelo Rodao 1995: 76),

(20) Vorbeugung (Brunnstein 1991: 6); Vorsorge/Früherkennung (ibid.: partie du titre); Viren-Vorsorge (ibid.: 111).

Les exemples (16) à (20) sont intéressants, car tous ne sont pas des métaphores. C'est le cas de prévenir et ses dérivés (< lat. praevenire/praeventio), ainsi que de l'allemand Vorsorge/Früherkennung $)^{13}$. Cependant, ces expressions sont typiques d'un discours fournissant de nombreuses métaphores, à savoir le discours médical: prévention ainsi que l'allemand Vorsorge évoquent tout de suite le contexte médical (voir la prévention contre le cancer, prévention du sida), et le mot composé Viren-Vorsorge suit le paradigme de Krebsvorsorge, qui est au coeur de la discussion sur la santé en Allemagne.

\footnotetext{
${ }^{13}$ Le mot prévention provient tout au plus historiquement d'une métaphore dont le locuteur contemporain n'est plus conscient.
} 
On retrouve donc des expressions non métaphoriques typiques du même discours source. C'est la raison pour laquelle il me semble plus adéquat de dire - comme je l'ai remarqué au début - que le discours sur le virus informatique emprunte certaines façons de parler des discours sources ${ }^{14}$.

Une des principales mesures préventives en médecine est la vaccination. Le concept qui y correspond est source d'expressions métaphoriques qui sont largement présentes dans les trois langues lorsqu'il est question de virus informatiques ${ }^{15}$.

\section{DISCOURS SOURCE 2: LA GUERRE}

Les contre-mesures sont également décrites en termes relevant du domaine de la guerre, ainsi en espagnol: combatir / luchar contra / hacer frente a:

(21) los virus se pueden combatir (PC actual, abril 1991: 222),

(22) luchar contra estos infecciones (ibid.: 221),

(23) hacer frente a esta amenaza (super, abril 1994: 26).

\section{DISCOURS SOURCE 3: LA BIOLOGIE}

Aux deux discours sources mentionnés s'ajoute un troisième, celui de la biologie. Je me contente de remarquer qu'on fait systématiquement appel à ce discours source pour certains sous-thèmes, par exemple pour décrire les caractéristiques des virus, le fait qu'ils se reproduisent et sont susceptibles de subir des mutations (pour plus de détails voir Sabban sous presse).

\section{DISCOURS SOURCES: RELATION DE DÉPENDANCE}

Dans une perspective diachronique, les trois discours sources ne sont pas sur le même plan; seuls le discours médical et le discours biologique donnent lieu à des innovations sur le plan métaphorique. Les expressions du discours guerrier, par

${ }^{14}$ Autres exemples de collocations empruntées aux discours sources biologique et médical et dont un (ou plusieurs) membres ne sont pas des métaphores: des combinaisons avec affecter et afectar, comme dans Le virus affecte le logiciel (La Recherche, oct. 1995: 24); los virus afectan todas las zonas del disco (cf. PC actual, abril 1994: 222). On passera ici sur le fait que les façons de parler plus complexes sont soit empruntées in toto, soit recombinées, d'où formation de collocations nouvelles. Pour une illustration, voir Sabban sous presse.

${ }^{15}$ Fr.: vaccination (internet I); vaccin (numérique) (PC Direct 10/1996: 138); esp.: vacuna (PC actual, abril 1991: 222; de Marcelo Rodao 1995: 78); vacunando al ordenador (de Marcelo Rodao 1995: 78); all.: Impfung (Brunnstein 1991: 115), impfen (ibid.: 113). Mise au point: les logiciels et le système d'exploitation peuvent être vaccinés à l'aide de pseudo-virus, qui simulent une infection devant l'imminence d'une contamination réelle (voir Brunnstein 1991: 113). La vaccination ne peut protéger que d'un virus connu (ibid.: 115). 
contre, sont déjà bien établies dans le discours médical: l'idée que les maladies sont des 'attaques contre' le corps humain et que celui-ci leur oppose des 'défenses', a donné diverses expressions dans les trois langues (cf. Dobrovol'skij 1997, Varela 1991). Il suffit de regarder quelques périphrases désignant le système immunitaire:

les défenses naturelles de l'organisme (L'Express 10.-16.10.1996: 38), defensas inmunitarias del organismo (Diccionario manual..., inmunitario), Abwehrkraft (Duden).

La métaphore de l'ennemi et de la guerre n'est donc pas une innovation dans le discours sur les anomalies programmées; elle est déjà disponible, elle est introduite par l'intermédiaire de la métaphore du virus. Schéma:

discours source: biologie
discours source: médecine
discours source: guerre d'innovations métaphoriques
métaphores déjà disponibles

\section{LA PERSPECTIVE DE L'AUTEUR DU TEXTE}

Qu'est-ce qui nous permet de dire que les discours sources décrits plus haut ne sont pas seulement une vue de l'esprit du linguiste, mais représentent aussi des catégories pertinentes pour l'auteur du texte? Cette question me permet de passer au problème de la formulation. La recherche consciente par l'auteur du texte de formulations s'adaptant à d'autres formulations présuppose, à mon avis, la pertinence de ces catégories.

Les comparaisons explicites entre discours source et discours cible ainsi que des remarques d'ordre métalinguistique dans le texte même en sont parmi les indices les plus nets:

(27) Un programme qui effectue une copie de lui-même est un virus, par analogie avec le mode d'action du virus biologique (PC Direct, oct. 1996: 139),

(28) C'est alors qu'ils [= ces virus] deviennent de parfaits analogues électroniques des virus biologiques (Le Nouvel Observateur 28/4 - 4/5/1994: 48 s.),

(29) Existe una gran similitud entre los virus que afectan al ser humano y los que aquejan a las máquinas (PC actual, abril 1991: 220),

(30) [...] los polimórficos, que sí aparentemente mutan. Y digo 'aparentemente', porque no es una mutación de tipo biológico, sino que [...]" (de Marcelo Rodao 1995: 70), 
Ähnlich wie die biologischen Verwandten brauchen auch die Computer-Viren einen Wirt, in diesem Fall eine ausführbare Programmdiskette (Bild der Wissenschaft 5/1992: 13),

(traduction: 'Tout comme les parents biologiques, les virus informatiques nécessitent la présence d'un hôte, dans ce cas une disquette de logiciel exécutable').

La fonction de ces comparaisons et commentaires est avant tout d'ordre explicatif; elles relèvent donc de la fonction plus large de la médiation de l'information.

J'aimerais revenir à l'exemple (31) que j'ai aussi traduit en français et qui contient la métaphore de l'hôte. Cet exemple confirme tout d'abord que l'auteur du texte a en tête le discours biologique comme source de la métaphore dans le contexte informatique. Du point de vue diachronique, le terme biologique hôte remonte, lui, à une métaphorisation de l'hôte humain. Mais pour l'auteur, le domaine biologique est le seul qui soit pertinent dans ce contexte, car le discours source biologique fournit nombre d'autres expressions au discours sur les virus informatiques. En d'autres termes, l'auteur effectue un choix, il sélectionne une isotopie en accord avec celle d'autres expressions.

Conclusion plus générale encore: Cet extrait montre que la description linguistique ne peut pas être assimilée simplement à des catégories conceptuelles opératoires pour l'auteur du texte, à savoir les catégories dont on peut supposer qu'elles interviennent dans le processus de formulation - comme le prétendent certains représentants de la linguistique cognitive. Ce qui est pertinent pour l'auteur dans cette activité ne peut pas être déterminé hors contexte.

\section{FONCTIONS DE LA MÉTAPHORE}

Comme je l'ai dit au début, les métaphores ont potentiellement une double fonction:

1. Première fonction: celle de la médiation d'une information nouvelle; en partant d'un concept connu, elles ouvrent une voie d'accès à la compréhension du phénomène et à la création d'un concept nouveau y correspondant. Cette fonction de la métaphore a fait l'objet d'expériences dans le domaine de la psychologie cognitive et de la didactique à plusieurs reprises, et je n'entre pas dans une discussion de cet aspect $\mathrm{ici}^{16}$.

2. Deuxième fonction: celle de la mise en scène de l'information. Cette seconde fonction nécessite l'activation d'autres dimensions sémantiques. La présence simultanée de plusieurs domaines sémantiques, les connotations qui y sont rattachées et les associations qui en résultent ont un effet de mise en scène qui peut varier selon les cas.

\footnotetext{
${ }^{16}$ Voir Gentner, Gentner 1983; Kieras, Bovair 1984; Simons 1984.
} 
Je me concentre dans ce qui suit sur les questions:

- Quels sont les procédés discursifs dont dispose l'auteur du texte pour activer ces autres dimensions sémantiques? (Voir 6.1.)

- Existe-t-il d'autres facteurs d'ordre non discursif pouvant intervenir dans la mise en scène de l'information? (Voir 6.2.)

\subsection{MISE EN SCÈNE DE L'INFORMATION: PROCÉDÉS DISCURSIFS}

Procédé 1: Actualisation du discours source dans le contexte immédiat

(32) Desde que el mundo es mundo, vida y enfermedad son conceptos encadenados. En algunos casos, el resultado final es la muerte. Y casi siempre, el culpable es un virus. Ahora han llegado a su ordenador. Los virus atacan a la máquinas (PC actual, abril 1991: 220).

Ce passage fait partie de l'introduction à un texte sur les virus informatiques. Le texte s'ouvre sur un ton pathétique, parlant de la vie, de la maladie et de la mort expériences fondamentales de la vie humaine. La transition de ce thème à celui de l'informatique s'effectue à l'intérieur de ce passage. La phrase soulignée est particulièrement intéressante à cet égard: le sujet du verbe han llegado, de par les règles de la cohésion textuelle, devrait être celui de la phrase précédente, donc les virus maladie. Du point de vue sémantique, par contre, ce sont les virus informatiques, sujet de la phrase suivante. Au moment où on passe - presque imperceptiblement - au véritable thème du texte, il y a donc forcément présence simultanée du discours source et du discours cible - ou croisement de deux isotopies (cf. la contribution de Ronald Landheer dans ce volume).

Procédé 2: Actualisation du discours source dans le contexte immédiat plus placement en position saillante.

\section{Titre: Windows 95 contracte son premier virus}

La mésaventure est de saison. Au moment où un froid polaire fait grelotter les Etats-Unis, Windows 95, le système d'exploitation des ordinateurs compatibles IBM [...], attrape son premier virus. Une fatalité dans la vie d'un logiciel, fût-il conçu par le géant américain Microsoft (Le Monde 6/2/96: 1).

On retrouve ici le même enchaînement thématique que dans l'exemple (32), c'està-dire qu'on passe du virus maladie au virus informatique. Au début du texte, paru en février, on se réfère à la saison, le temps glacial suggérant les affections grippales qu'on attrape en hiver. L'expression la vie (d'un logiciel) rappelle le discours source évoqué au début de l'article. En plus, on peut observer ici l'insertion d'une métaphore (contracter) dans le titre. Les titres sont généralement le lieu privilégié de formulations équivoques ou tout simplement marquées attirant l'attention du lecteur et suscitant sa curiosité. 
Ces remarques confirment l'hypothèse que les métaphores ont été choisies dans un but de mise en scène. Un dernier indice, mais non des moindres, est que la métaphore contracter n'apparaît pas dans le reste du corpus dans ce contexte précis.

L'actualisation du discours source peut s'effectuer également par le moyen d'une illustration. Ainsi on trouve, dans un numéro du magazine PCmag $\left(n^{\circ} 111,1997\right)$, deux illustrations en position saillante. L'une, évoquant le discours source de la médecine (on y voit une dizaine de comprimés et cachets), figure dans la table des matières, à côté du titre d'un dossier sur les antivirus (p. 6); l'autre, évoquant le discours source de la biologie, est placée au début du dossier même (p. 124-25: des insectes qui sortent d'un ordinateur en brisant la vitre de l'écran).

Procédé 3: Actualisation du discours source par la disposition des textes et des thèmes à l'intérieur d'un périodique.

La même succession de thèmes que dans les exemples précédents (voir (32) et (33)) est vérifiable à un niveau supérieur, à savoir au niveau des textes dans leur ensemble. Ainsi, dans un numéro du périodique Sciences et Avenir (février 1994) on trouve deux textes sur les virus: le premier traite d'un virus mortel récemment découvert. Une illustration montre le porteur de ce virus, une souris photographiée de nuit, en gros plan (p. 62). La photo et le titre Le tueur d'Albuquerque évoquent un contexte de criminalité, le passage au-dessous du titre parle du virus porteur de la maladie mortelle. A la page suivante, il y a le deuxième texte au sujet des virus informatiques. Il suffit donc de feuilleter le périodique de façon superficielle pour faire le lien entre discours source et discours cible. Tous deux sont alors très certainement superposés par association. Ce procédé suscite des émotions élémentaires, le reportage se rapproche de la presse à sensation.

\section{Procédé 4: Création de métaphores nouvelles.}

Un des procédés classiques de 'mise en scène' de l'information est l'emploi de métaphores nouvelles. Celles-ci ont toujours une forte chance d'évoquer leur domaine source. Tous les discours sources mentionnés constituent des réservoirs dans lesquels l'auteur peut puiser des métaphores nouvelles ${ }^{17}$. Mais, dans quels cas peut-on assumer à juste titre qu'il s'agit de métaphores nouvelles?

1) dans le cas où les images utilisées sont particulièrement frappantes par rapport au sujet du texte. Ainsi on trouve, dans deux textes français, une accumulation d'i-

\footnotetext{
${ }^{17}$ La notion de «frame» (Fillmore 1985) peut être utilisée pour saisir les relations entre les métaphores d'un même champ. Selon Fillmore, les métaphores peuvent être des «[...] lexical representatives of some single coherent schematization of experience or knowledge. [...] What holds such word groups together is the fact of their being motivated by, founded on, and co-structured with, specific unified frameworks of knowledge, or coherent schematizations of experience [...].» (Fillmore 1985: 223; c'est moi qui souligne).
} 
mages anachroniques tirées de la guerre au moyen âge - citadelle, rempart, se lancer à l'assaut de (voir les exemples ci-dessous) - , alors qu'il s'agit d'une forme éminemment moderne d' 'agression' (voir cette agression moderne, exemple (11)):

(34) Windows 95 n'est plus la citadelle imprenable qu'il était encore l'an dernier (Lilen 1996: XIV),

(35) on croyait que Windows 95 constituerait un bon rempart (ibid.: XIV),

(36) Titre de l'article: Un PC imprenable (PC Direct 10/1996: 141),

(37) quand un nouveau virus se lance à l'assaut du parc informatique mondial (PC Direct 10/1996: 141).

2) lorsque les expressions métaphorisées sont marquées stylistiquement:

Un seul exemple, allemand, qui constitue le titre d'un article:

Wenn der Computer krank feiert (Bild der Wissenschaft 5/1992: 13).

\subsection{MISE EN SCÈNE DE L'INFORMATION: FACTEUR NON DISCURSIF}

Pour terminer, je voudrais aborder un facteur non discursif jouant un rôle dans l'évocation d'une autre dimension sémantique. Il s'agit de la forme des mots qui peut intervenir dans l'interprétation.

Cet aspect est particulièrement net en allemand. Il est bien connu qu'en allemand il y a beaucoup de paires de lexèmes dont l'un est d'origine germanique, l'autre d'origine latino-romane - exemple: (sich) anstecken à côté de (sich) infizieren. Le choix de l'un ou de l'autre est conditionné par des paramètres socioculturels et contextuels.

On pourrait penser que ces mots, étant synonymes au niveau conceptuel (cf. Leech 1974), peuvent être soumis au même processus de métaphorisation, c'est-à-dire être employés tous deux de façon métaphorique dans le discours sur le virus informatique. Or, il n'en est rien. Les mots d'origine germanique sont régulièrement accompagnés d'un effet d'actualisation du domaine source et, donc, d'un effet de mise en scène de l'information. A titre d'illustration, voir les effets différents produits par les paires de phrases suivantes:

(39a) Die Gefährlichkeit der Viren ist verschieden,

(39b) Die Gefährlichkeit der «Erregen» ist verschieden (Lübecker Nachrichten 8/6/1996: 5; avec des guillemets comme signe de distanciation),

(40a) Der Virus hat alle PC-s infiziert (cf. exemples (7) et (8)),

(40b) Der Virus hat alle PC-s angesteckt (exemple hypothétique),

(41a) Der Michelangelo-Virus attackiert die Festplatte (Lübecker Nachrichten 8/6/96: 5),

(41b) «Computer-Viren greifen die Hardware an!» (Titre d'article, cité dans Brunnstein 1991: 9). 
Explication: Les mots d'origine germanique (voir exemples (b)) sont plus transparents que leurs 'homologues' latino-romans. C'est sans doute à cause de cette transparence que l'acception littérale s'impose à la perception. A l'inverse, les mots d'origine latino-romane (voir exemples (a)) ont d'emblée un effet de distanciation, dans une langue comme l'allemand. Leur analyse sémantique reste plus superficielle. On peut faire le rapprochement avec un autre contexte, à savoir l'utilisation d'éléments étrangers dans les noms de produits. Dans de nombreux cas, seuls les noms formés à l'aide de composants étrangers (ou qui en ont l'air) sont considérés comme acceptables sur le marché; les 'traductions germanisantes' de ces mêmes noms seraient perçues comme trop directes et même peu sérieuses (Platen 1997: 51). Que l'on compare, à titre d'exemple, le nom latinisant Tussafug, un médicament pour la toux, avec son homologue germanisé Husteflucht (ibid.).

Ces dernières remarques ne me font m'écarter qu'en apparence du sujet central de mon exposé. En dehors de la conclusion générale que «tout n'est pas discursif dans la langue», ces dernières observations peuvent être importantes pour le traducteur. Elles lui fournissent une piste pour bien saisir, dans la phase de la compréhension du texte à traduire, les effets produits par le choix des expressions ainsi que pour trouver, dans la langue cible, des moyens qui soient équivalents au niveau de l'effet produit.

\section{ANNEXE: TEXTES EXPLOITÉS}

\section{Français}

Alberganti, M., Windows contracte son premier virus, in: Le Monde, 6/2/1996, 1.

G ru hi er, F., Ces virus qui ravagent nos puces, in: Le Nouvel Observateur, 28/4 - 4/5/1994, 48-50.

Guide d'achat - Les antivirus (1: Vaccin numérique: immunisez votre PC; 2: Procédures d'évaluation), in: PC Direct. Le magazine des acheteurs, $\mathrm{n}^{\circ} 47$, oct. 1996, 138-151.

La faute d'un 'probable virus informatique', in: Le Monde, 3/2/1996, 8.

Lilen, H., Virus - Mode d'emploi. Prévention, Détection, Désinfection, Paris et al. 1996.

Thfoin, M., Déjouez les macrovirus! In: PCmag, ${ }^{0}$ 111, juin 1997, 124-134.

Un monde sans virus?, Internet, http://www.tdi-service.fr/Page4.htm., mars 1997.

\section{Espagnol}

G andía, J., Endemoniados bacilos patrios, in: PC actual, abril 1991, 220-222.

De Marcelo Rodao, J., Guía de campo de los virus informáticos, Madrid 1995.

Menéndez, E., Limpia, fija y da esplendor, in: Super. Revista para los usuarios de ordenadores personales, $\mathrm{n}^{\circ} 11$, abril 1994, 26-27.

\section{Allemand}

Brunnstein, K., Computer-Viren-Report. Gefahren - Wirkung - Aufbau - Früherkennung - Vorsorge, 2ème éd.. actual. et ampl., Planegg 1991, 5-70, 103-119.

Vieten, M., Wenn der Computer krank feiert, in: Bild der Wissenschaft, 5/1992, 13.

Helfer im Netz, in: Bild der Wissenschaft, 3/1994, 12.

Mehr als 9000 Viren bedrohen die Computer-Welt, in: Lübecker Nachrichten, 8/6/1996, 5. 


\section{BIBLIOGRAPHIE}

B iere, B. U., Li ebert, W.-A. (éd.) (1997), Metaphern, Medien, Wissenschaft. Zur Vermittlung der AIDS-Forschung in Presse und Rundfunk, Opladen.

Diccionario manual e ilustrado de la lengua española, $\left({ }^{4} 1989\right)$, Madrid.

Dobrovol'skij, D. (1997), Metaphernmodelle und Idiome in mündlichen Fach- und Vermittlungstexten. Eine exemplarische Analyse zum Thema AIDS, dans: Biere, B. U., Liebert, W.-A. (éd.), 148-179.

Duden - Das große Wörterbuch der deutschen Sprache in acht Bänden. 2., völlig neu bearb. u. erw. Aufl., Mannheim et al. 1995.

Fernández-Sánchez, M. (1990), Comprender e interpretar un discurso de imitación sobre el discurso de divulgación científica, dans: Le langage et l'homme 25: 284-291.

Fill more, Ch. J. (1985), Frames and the Semantics of Understanding, dans: Quaderni di semantica VI. 2, 222-254.

Gentner, D., Gentner, D. R. (1983), Flowing Waters or Teeming Crowds: Mental Models of Electricity, dans: Gentner, D., Stevens, A. L. (éd.), 99-129.

Gentner, D., Stevens, A. L. (éd.) (1983), Mental Models, Hillsdale, N.J. et al.

Jeanneret, Y. (1994), Ecrire la Science. Formes et enjeux de la vulgarisation, Paris.

Kieras, D., Bovair, S. (1984), The Role of a Mental Model in Learning to Operate a Device, dans: Cognitive Science 8: 255-273.

Koller, W: ( $\left.{ }^{4} 1992\right)$, Einführung in die Übersetzungswissenschaft, Heidelberg / Wiesbaden.

Lak off, G. (1987), Women, Fire, and Dangerous Things. What Categories Reveal about the Mind, Chicago-London.

Lak off, G., Johns on, M. (1980), Metaphors We Live By, Chicago-London [trad. française: Les métaphores dans la vie quotidienne, Paris 1985].

Landheer, R. (éd.) (1994), Les figures de rhétorique et leur actualité en linguistique, Langue française 101 .

Leech, G. N. (1974), Semantics, Harmondsworth.

Loffler-La urian, A.-M. (1994), Réflexions sur la métaphore dans les discours scientifiques de vulgarisation, dans: Langue française 101, 72-79.

Moirand, S. et al. (éd.) (1994), Parcours linguistiques de discours spécialisés. Colloque en Sorbonne les 23-24-25 septembre 1992, Bern et al.

Mortureux, M.-F. (1994), Comment peut-on définir la propriété d'un mot? Dans: Moirand, Sophie et al. (éd.), 3-10.

Platen, Ch. (1997), 'Ökonymie'. Zur Produktnamenlinguistik im Europäischen Binnenmarkt, Tübingen.

Sabban, A. (sous presse), Metaphernfelder in Texten zum Computervirus - Eine vergleichende Untersuchung zu den Sprachen Deutsch, Französisch und Spanisch. Dans: Börner, Vogel (éd.), Kontrast und Äquivalenz. Tübinger Beiträge zur Linguistik, Tübingen.

Simons, P.R.J. (1984), Instructing With Analogies, dans: Journal of Educational Psychology 76: 513-527.

V are la, F. J. (1991), Der Körper denkt. Das Immunsystem und der Prozeß der Körper-Individuierung, in: Gumbrecht, H. U., Pfeiffer, K. L. (éd.), Paradoxien, Dissonanzen, Zusammenbrüche. Situationen offener Epistemologie, Frankfurt/Main, 727-743.

We inrich, H. (1976), Münze und Wort. Untersuchungen an einem Bildfeld, dans: Weinrich, H., Sprache in Texten, Stuttgart, 276-290. 